\title{
Estimates of probabilistic widths of the diagonal operator of finite-dimensional sets with the Gaussian measure
}

\author{
Jiehua Zhou' and Yuewu $\mathrm{Li}^{2 *}$
}

\section{${ }^{*}$ Correspondence:}

yuewul@126.com

${ }^{2}$ School of Mathematical Sciences,

Hulunbeier University, Hulunbeier,

Inner Mongolia 021008, China

Full list of author information is

available at the end of the article

\begin{abstract}
In this paper, we estimate the asymptotic orders of probabilistic and average widths of the compact embedding operators from the Sobolev space $W_{2}^{r}(\mathbb{T})$ into $L_{q}(\mathbb{T})$

$(1 \leq q \leq \infty)$ with the Gaussian measure.

MSC: 41A10; 41A46; 42A61; 46C99
\end{abstract}

Keywords: probabilistic width; average widths; Sobolev space; Gaussian measure

\section{Introduction and main results}

Problems of $n$-widths in the approximation theory have by now been studied in depth. A great deal of classical problems have been solved, and interesting new developments have appeared. For example, the problems of probabilistic, average and stochastic widths, which can reflect the behavior of function on the whole class and give information about the measure of the elements in the class that can be approximated to this or that degree, are the problems of this kind. For the results related to the probabilistic, average and stochastic widths, the reader may be referred to Sul'din [1, 2], Traub et al. [3], Maiorov [4-7], Mathé [8-12], Sun [13, 14], and Ritter [15]. The new developments in this direction can be found in Fang's papers [16-21]. Moreover, Carl and Pajor [22] proved an inequality with respect to the Gelfand numbers of an operator $u$ from $\ell_{1}^{N}$ into a Hilbert space, from which one can immediately derive the inequality related to the Kolmogorov numbers by the known duality. In this article we continue the previous works and prove the estimates of probabilistic widths of the diagonal operators from $\mathbb{R}^{m}$ onto $\ell_{q}^{m}$.

First, we recall some useful concepts. Let $W$ be a bounded subset of a normed linear space $X$ with the norm $\|\cdot\|_{X}$, and $F_{N}$ be an $N$-dimensional subspace of $X$. The quantity

$$
e\left(W, F_{N}, X\right)=\sup _{x \in W} e\left(x, F_{N}, X\right)
$$

where

$$
e\left(x, F_{N}, X\right)=\inf _{y \in F_{N}}\|x-y\|_{X}
$$

(0) 2013 Zhou and Li; licensee Springer. This is an Open Access article distributed under the terms of the Creative Commons Attribution License (http://creativecommons.org/licenses/by/2.0), which permits unrestricted use, distribution, and reproduction in any medium, provided the original work is properly cited. 
is called the deviation of $W$ from $F_{N}$. It shows how well the 'worst' elements of $W$ can be approximated by $F_{N}$. The number

$$
d_{N}(W, X)=\inf _{F_{N}} e\left(W, F_{N}, X\right)=\inf _{F_{N}} \sup _{x \in W} \inf _{y \in F_{N}}\|x-y\|_{X},
$$

where $F_{N}$ runs through all possible linear subspaces of $X$ of dimension at most $N$, is called the Kolmogorov's $N$-width of $W$ in $X$. Assume that $W$ contains a Borel field $\mathcal{B}$ consisting of open subsets of $W$ and equipped with a probabilistic measure $\mu$ defined on $\mathcal{B}$. That is, $\mu$ is a $\sigma$-additive nonnegative function on $\mathcal{B}$, and $\mu(W)=1$. Let $\delta \in[0,1]$ be an arbitrary number. The corresponding probabilistic Kolmogorov's $(N, \delta)$-width of a set $W$ with a measure $\mu$ in the space $X$ is defined by

$$
d_{N, \delta}(W, \mu, X)=\inf _{G_{\delta}} d_{N}(W \backslash G, X),
$$

where $G_{\delta}$ runs through all possible subsets in $\mathcal{B}$ with measure $\mu\left(G_{\delta}\right) \leq \delta$. The $p$-average Kolmogorov's $N$-width is defined by

$$
d_{N}^{(a)}(W, \mu, X)_{p}=\inf _{F_{N}}\left(\int_{W} e\left(x, F_{N}, X\right)^{p} d \mu(x)\right)^{1 / p}, \quad 0<p<\infty
$$

where $F_{N}$ in (2) runs over all linear subspaces of $X$ of dimension at most $N$. Let $\ell_{p}^{m}$ be an $m$-dimensional normed space of vectors $x=\left(x_{1}, \ldots, x_{m}\right) \in \mathbb{R}^{m}$, with a norm

$$
\|x\|_{\ell_{p}^{m}}= \begin{cases}\left(\sum_{i=1}^{m}\left|x_{i}\right|^{p}\right)^{1 / p}, & 1 \leq p<\infty \\ \max _{1 \leq i \leq m}\left|x_{i}\right|, & p=\infty\end{cases}
$$

Consider in $\mathbb{R}^{m}$ the standard Gaussian measure $v=v_{m}$, which is defined as

$$
v(G)=(2 \pi)^{-m / 2} \int_{G} \exp \left(-\frac{1}{2}\|x\|_{2}^{2}\right) d x,
$$

where $G$ is any Borel subset in $\mathbb{R}^{m}$. Obviously, $v\left(\mathbb{R}^{m}\right)=1$.

Denote by $B_{p}^{m}(\rho)=\left\{x \in \ell_{p}^{m}:\|x\|_{p} \leq \rho\right\}$ the ball of radius $\rho$ in $\ell_{p}^{m}$. Let $B_{p}^{m}=B_{p}^{m}(1)$.

Let $N=0,1, \ldots, \delta \in[0,1)$ be arbitrary and $T_{m}$ be a linear invertible operator from $\mathbb{R}^{m}$ onto $\ell_{q}^{m}$. We define the probabilistic $(N, \delta)$-width of the operator acting in space $\mathbb{R}^{m}$ equipped with the Gaussian measure $v$ in $\ell_{q}^{m}$-norm:

$$
d_{N, \delta}\left(T_{m}: \mathbb{R}^{m} \rightarrow \ell_{q}^{m}, v\right)=\inf _{G} \inf _{\mathcal{L}_{N}} e\left(T_{m}\left(\mathbb{R}^{m} \backslash G\right), \mathcal{L}_{N}, \ell_{q}^{m}\right),
$$

where $v(G)<\delta, \operatorname{dim} \mathcal{L}_{N} \leq N$.

Maiorov in [6] proved the following result.

Theorem A [6] For $m>2 N, \delta \in(0,1 / 2], 1 \leq q \leq 2$, then

$$
d_{N, \delta}\left(\mathbb{R}^{m}, \ell_{q}^{m}, v\right) \asymp m^{\frac{1}{q}-\frac{1}{2}} \sqrt{m+\ln (1 / \delta)} .
$$

In [22], Carl and Pajor proved the following result with respect to Gelfand numbers of an operator with values in a Hilbert space. 
Theorem B [22] Let $T$ be an operator from $\ell_{1}^{m}$ into a Hilbert space $H$. Then

$$
d^{N}(T) \leq C\left(\frac{\log \left(\frac{m}{N}+1\right)}{N}\right)^{1 / 2}\|T\|
$$

for $1 \leq N \leq m, m=1,2, \ldots$, where $C>0$ is a universal constant.

Detailed facts about the usual widths, such as the Kolmogorov's $N$-widths and $N$ th Gelfand numbers (or Gelfand $N$-widths) of $T$ were given in the books [23-26].

\section{Remark 1}

(a) Theorem A shows the asymptotic expression of the probabilistic widths of the identity embedding from $\mathbb{R}^{m}$ into $\ell_{q}^{m}, 1 \leq q \leq 2$.

(b) Theorem B gives the upper estimate of Gelfand numbers of operators from $\ell_{1}^{m}$ into a Hilbert space, and some of its striking applications in the geometry of Banach spaces and Rademacher processes can be found in [22]. By the dual relation, it is easy to obtain the similar upper estimate of the Kolmogorov's $N$-widths $d_{N}(T)$ of operators from $\ell_{2}^{m}$ into $\ell_{\infty}^{m}$, i.e.,

$$
d_{N}(T) \leq C\left(\frac{\log \left(\frac{m}{N}+1\right)}{N}\right)^{1 / 2}\|T\|
$$

(c) Motivated by Theorems A and B, in general cases, here we investigate the asymptotic estimate of probabilistic widths for diagonal operators from $\mathbb{R}^{m}$ onto $\ell_{q}^{m}$, $1 \leq q \leq \infty$.

Now we are in a position to formulate our main results.

Theorem 1 For $m>N, \delta \in(0,1 / 2]$, then

$$
d_{N, \delta}\left(T_{m}: \mathbb{R}^{m} \rightarrow \ell_{\infty}^{m}, v\right) \leq C\left\|T_{m}\right\| \sqrt{(1+(1 / N) \ln (1 / \delta)) \ln (e m / N)} .
$$

Theorem 2 For $m>2 N, \delta \in(0,1 / 2]$, then

$$
d_{N, \delta}\left(T_{m}: \mathbb{R}^{m} \rightarrow \ell_{1}^{m}, v\right) \geq C^{\prime}\left\|T_{m}\right\| \sqrt{m+\ln (1 / \delta)} .
$$

\section{Proof of main results}

In order to prove Theorems 1 and 2, we also need some auxiliary assertions.

Lemma 1 Let $\delta \in(0, \sqrt{2 / e \pi}]$, and let $T_{m}$ be a bounded linear invertible operator from $\mathbb{R}^{m}$ onto $\ell_{\infty}^{m}$. Then, for any vector $z \in \mathbb{R}^{m}$,

$$
v\left(x:\left|\left(T_{m} x, z\right)\right| \geq 2\left\|T_{m}\right\| \sqrt{\ln (1 / \delta)}\|z\|_{2}\right) \leq \delta .
$$

Proof First, assume that $T_{m}$ is a diagonal operator of $\mathbb{R}^{m}$, i.e., $T_{m} x=\left(\lambda_{i} x_{i}\right)_{i=1}^{m}$, for any $x \in \mathbb{R}^{m}$. Without loss of generality, assume that the sequence of the absolute of eigenvalues $\lambda_{i}, i=1, \ldots, m$, is arranged non-increasingly, i.e., $\left|\lambda_{1}\right| \geq\left|\lambda_{2}\right| \geq \cdots \geq\left|\lambda_{m}\right|>0$. It is 
known that

$$
\left\|T_{m}\right\|=\max _{1 \leq i \leq m}\left|\lambda_{i}\right|=\left|\lambda_{1}\right|
$$

Since $v$ is invariant with respect to orthogonal transformation of $\mathbb{R}^{m}$, it suffices to prove the lemma for the vector $z^{*}=\left(\|z\|_{2}, 0, \ldots, 0\right)$. Let $\varepsilon \in\left(0, e^{-1}\right]$ be arbitrary. We have

$$
\begin{aligned}
v( & \left.x:\left|\left(T_{m} x, z^{*}\right)\right| \geq\left\|T_{m}\right\| \sqrt{\ln (1 / \varepsilon)}\|z\|_{2}\right) \\
& =v\left(x:\left|\lambda_{1} x_{1}\right|\|z\|_{2} \geq\left|\lambda_{1}\right| \sqrt{\ln (1 / \varepsilon)}\|z\|_{2}\right) \\
& =v\left(x:\left|x_{1}\right| \geq \sqrt{\ln (1 / \varepsilon)}\right) \\
& =\frac{2}{\sqrt{\pi}} \int_{\sqrt{(1 / 2) \ln (1 / \varepsilon)}}^{\infty} \exp \left(-t^{2}\right) d t \leq\left(\frac{2 \varepsilon}{\pi}\right)^{1 / 2} .
\end{aligned}
$$

Here we use the inequality

$$
\int_{u}^{\infty} \exp \left(-t^{2}\right) d t<\frac{1}{2 u} \exp \left(-u^{2}\right), \quad u \geq \frac{1}{\sqrt{2}} .
$$

From (3) we obtain the assertion of the lemma by $\delta=\sqrt{2 \varepsilon / \pi}$.

Next, assume that $T_{m}$ is a symmetric transformation of $\mathbb{R}^{m}$, then there is an orthogonal matrix $U$ of order $m$ such that the matrix $U T_{m} U^{T}$ is a diagonal matrix. Since the Gaussian measure is invariant for orthogonal transformation, the result holds for symmetric transformation $T_{m}$.

Finally, assume that $T_{m}$ is a general invertible linear transformation from $\mathbb{R}^{m}$ onto $\mathbb{R}^{m}$, then there are two matrices $U$ and $S$ such that $T_{m}=U S$, where $U$ is an orthogonal matrix and $S$ is a positive definite symmetric matrix. As the same reason above, the result holds for the transformation $T_{m}$.

Thus Lemma 1 is proved.

The following inequality will be used (see [27]). For any integers $N$ and $m$ with $m>N \geq$ 1 , there exists a subspace $H$ of $\mathbb{R}^{m}$ of dimension $\operatorname{dim} H \geq m-N$ such that for any $x \in H$,

$$
\|x\|_{2} \leq c_{0}\left(\frac{\ln (e m / N)}{N}\right)^{1 / 2}\|x\|_{1}
$$

where $c_{0}$ is an absolute constant.

Let $G \subset \mathbb{R}^{m}$ be a set. We introduce in $\mathbb{R}^{m}$ another norm for the operator $T_{m}: \mathbb{R}^{m} \rightarrow \ell_{\infty}^{m}$ :

$$
\|x\|_{G}=\sup _{y \in \mathbb{R}^{m} \backslash G}\left|\left(T_{m} y, x\right)\right|
$$

Lemma 2 For any $\delta \in(0,1 / 2]$ and an arbitrary operator $T_{m}$ from $\mathbb{R}^{m}$ onto $\ell_{\infty}^{m}$, there exists a subset $G=G_{\delta}$ of $\mathbb{R}^{m}$ with measure $v(G) \leq \delta$ such that

$$
\sup _{z \in B_{1}^{m} \cap H}\|z\|_{G} \leq c_{1}\left\|T_{m}\right\|\left(\frac{1}{N} \ln \frac{\exp (a N)}{\delta} \ln \frac{e m}{N}\right)^{1 / 2}
$$

where $a$ and $c_{1}$ are absolute constants. 
Proof Let $k=[8(N+1) / \ln (e m / N)]$ and consider the $k^{-1}$-net

$$
S=\left\{\left(s_{1} / k, \ldots, s_{m} / k\right): s_{1}, \ldots, s_{m} \in \mathbb{Z},\left|s_{1}\right|+\cdots+\left|s_{m}\right| \leq k\right\}
$$

for the $B_{1}^{m}$ in $\ell_{\infty}^{m}$-norm. Using the inequality $\left(\begin{array}{c}m \\ \ell\end{array}\right) \leq\left(\frac{e m}{\ell}\right)^{\ell}$, we estimate the cardinality of $S$ :

$$
\operatorname{card} S \leq \sum_{\ell=1}^{k} 2^{\ell}\left(\begin{array}{c}
m+\ell \\
\ell
\end{array}\right) \leq \sum_{\ell=1}^{k} 2^{\ell}\left(\frac{4 e m}{\ell}\right)^{\ell} \leq\left(\frac{8 e m}{k}\right)^{k} \leq e^{a N}
$$

where $a$ is some absolute constant.

Consider the polyhedron $Q=B_{1}^{m} \cap k^{-1} B_{\infty}^{m}$. Let $Q^{\prime}$ be the set of extremal points of $Q$. The set $Q^{\prime}$ consists of vectors with $k$ coordinates equal to $\pm k^{-1}$ and the remaining coordinates zero. This implies that $Q^{\prime} \subset S$, and hence card $Q^{\prime} \leq \exp (a N)$.

Let $\varepsilon=\delta / \exp (a N)$. In $\mathbb{R}^{m}$ we consider the set $G=\bigcup_{s \in S} G_{s}$, where

$$
G_{s}=\left\{y \in \mathbb{R}^{m}:\left|\left(T_{m} y, s\right)\right| \geq 2\left\|T_{m}\right\| \sqrt{\ln (1 / \varepsilon)}\|s\|_{2}\right\} .
$$

Let $z \in B_{1}^{m} \cap H$ be any point, and $s \in S$ be a point closest to $z$ in $\ell_{\infty}^{m}$-norm. Then $z=s+t$ for some $t \in Q$. From Lemma 1,

$$
\|z\|_{G} \leq\|s\|_{G}+\|t\|_{G} \leq 2\left\|T_{m}\right\| \sqrt{\ln (1 / \varepsilon)}\|s\|_{2}+\|t\|_{G} .
$$

Using the definition of $Q$, we have $\|t\|_{2}^{2} \leq\|t\|_{1}\|t\|_{\infty} \leq k^{-1}$. From this and the definition of $Q^{\prime}$ and $G$,

$$
\begin{aligned}
\|t\|_{G} & \leq \max _{t \in Q^{\prime}}\|t\|_{G} \leq \max _{t \in S \cap Q}\|t\|_{G} \\
& \leq 2\left\|T_{m}\right\| \sqrt{\ln (1 / \varepsilon)} \max _{t \in S \cap Q}\|t\|_{2} \leq 2\left\|T_{m}\right\| \sqrt{k^{-1} \ln (1 / \varepsilon)} .
\end{aligned}
$$

Therefore from (6),

$$
\begin{aligned}
\|z\|_{G} & \leq 2\left\|T_{m}\right\| \sqrt{\ln (1 / \varepsilon)}\left(\|z\|_{2}+\|t\|_{2}\right)+\|t\|_{G} \\
& \leq 2\left\|T_{m}\right\| \sqrt{\ln (1 / \varepsilon)}\left(\|z\|_{2}+2 k^{-1 / 2}\right) .
\end{aligned}
$$

Since $z \in H$, it follows from the inequalities (4) and (7) that

$$
\|z\|_{G} \leq c_{1}\left\|T_{m}\right\| \sqrt{k^{-1} \ln (1 / \varepsilon)} \leq c_{2}\left\|T_{m}\right\| \sqrt{(1 / N) \ln (1 / \varepsilon) \ln (e m / N)} .
$$

Using Lemma 1 and the inequality (5), we can estimate the measure of G:

$$
v(G) \leq \sum_{s \in S} v\left(G_{s}\right) \leq \varepsilon \operatorname{card} S \leq \varepsilon \exp (a N)=\delta .
$$

Thus, Lemma 2 is proved. 
Proof of Theorem 1 Using the duality in $\mathbb{R}^{m}$ and Lemma 2, we have

$$
\begin{aligned}
\sup _{x \in \mathbb{R}^{m} \backslash G \in} \inf _{y \in H^{\perp}}\left\|T_{m} x-y\right\|_{\infty} & =\sup _{x \in \mathbb{R}^{m} \backslash G \in G \in H \cap B_{1}^{m}}\left|\left(T_{m} x, z\right)\right| \\
& =\sup _{z \in H \cap B_{1}^{m}}\|z\|_{G} \leq c\left\|T_{m}\right\| \sqrt{(1 / N) \ln (1 / \varepsilon) \ln (e m / N)} \\
& =c\left\|T_{m}\right\| \sqrt{(1 / N) \ln (\exp (a N) / \delta) \ln (e m / N),}
\end{aligned}
$$

where $H^{\perp}$ is the orthogonal complement of $H$ and $\operatorname{dim} H^{\perp} \leq N$. The proof of Theorem 1 is completed.

Let us proceed to the proof of Theorem 2. For this, we first prove four lemmas. We introduce a definition. For arbitrary $\varepsilon>0$, the $\varepsilon$-cardinality of a subset $K$ of $\ell_{1}^{m}$ is defined to be

$$
\mathcal{N}_{\varepsilon}(K)=\min \left\{\mathcal{N}: z_{1}, \ldots, z_{\mathcal{N}} \in \mathbb{R}^{m}, e\left(K,\left\{z_{1}, \ldots, z_{\mathcal{N}}\right\}\right) \leq \varepsilon\right\}
$$

where

$$
e\left(K,\left\{z_{1}, \ldots, z_{\mathcal{N}}\right\}\right)=\sup _{x \in K} \min _{i=1, \ldots, \mathcal{N}}\left\|x-z_{i}\right\|_{1}
$$

is the deviation of $K$ from the set $\left\{z_{1}, \ldots, z_{\mathcal{N}}\right\}$ in $\ell_{1}^{m}$.

Let $\lambda$ be a Lebesgue measure in $\mathbb{R}$, normalized by the condition $\lambda(B)=1$, where $B=B_{2}^{m}$. We consider Kolmogorov's $(N, \delta)$-width of the ball $B$ with measure $\lambda$ in the $\ell_{1}^{m}$-norm:

$$
d_{N, \delta} \equiv d_{N, \delta}\left(T_{m}: B \rightarrow \ell_{1}^{m}, \lambda\right)=\inf _{G} \inf _{\mathcal{L}} e\left(T_{m}(B \backslash G), \mathcal{L}, \ell_{1}^{m}\right),
$$

where the $T_{m}$ is as above, the infima are over all possible subsets $G \subset B$ of measure $\lambda(G) \leq$ $\delta$ and all subspaces $\mathscr{L} \subset \mathbb{R}^{m}$ with $\operatorname{dim} \mathscr{L} \leq N$.

Lemma 3 Suppose that $D \subset B$ is an arbitrary subset with measure $\lambda(D) \leq \delta$. Then, for any $\varepsilon>d_{N, \delta}$,

$$
\mathcal{N}_{\varepsilon}\left(T_{m}(B \backslash D)\right) \leq\left(1+\frac{4\left\|T_{m}\right\|}{\varepsilon-d_{N, \delta}}\right)^{m} .
$$

Proof Let $h>0$ be any number, and let $H$ be any subspace of $\mathbb{R}$ with $\operatorname{dim} H \leq N$ such that

$$
e\left(T_{m}(B \backslash D), H, \ell_{1}^{m}\right)-h \leq d_{N, \delta} .
$$

Let $\varepsilon^{\prime}=\varepsilon-d_{N, \delta}$. We consider the set $Q=2\left(T_{m}(B \backslash D)\right) \cap H$. Let $Q_{\varepsilon^{\prime}}=\left\{z_{1}, \ldots, z_{\mathcal{N}}\right\}$ be the maximal subset of $Q$ such that $\left\|z_{i}-z_{j}\right\|_{1} \geq \varepsilon^{\prime}$ for all $i \neq j$. Clearly, by maximality $Q_{\varepsilon^{\prime}}$ is a $\varepsilon^{\prime}$-net of $Q$ for $\|\cdot\|_{1}$. The balls $z_{i}+\left(\varepsilon^{\prime} / 2\right) B_{1}^{m}$ are disjoint and all contained in $Q+\left(\varepsilon^{\prime} / 2\right) B_{1}^{m}$. Therefore, taking volumes we can obtain

$$
\sum_{i=1}^{\mathcal{N}} \operatorname{vol}\left(z_{i}+\left(\varepsilon^{\prime} / 2\right) B_{1}^{m}\right) \leq \operatorname{vol}\left(Q+\left(\varepsilon^{\prime} / 2\right) B_{1}^{m}\right) \text {. }
$$


Hence, we have

$$
\mathcal{N} \cdot\left(\varepsilon^{\prime} / 2\right) \operatorname{vol}\left(B_{1}^{m}\right) \leq \operatorname{vol}\left(Q+\left(\varepsilon^{\prime} / 2\right) B_{1}^{m}\right)
$$

By $Q=2\left(T_{m}(B \backslash D)\right) \cap H \subset 2 T_{m}(B) \subset 2 T_{m}\left(B_{1}^{m}\right) \subset 2\left\|T_{m}\right\|\left(B_{1}^{m}\right)$ and (9), we have

$$
\mathcal{N}\left(\varepsilon^{\prime} / 2\right)^{m} \operatorname{vol}\left(B_{1}^{m}\right) \leq\left(2\left\|T_{m}\right\|+\left(\varepsilon^{\prime} / 2\right)\right)^{m} \operatorname{vol}\left(B_{1}^{m}\right)
$$

that is,

$$
\mathcal{N} \leq\left(1+\frac{4\left\|T_{m}\right\|}{\varepsilon^{\prime}}\right)^{m} .
$$

Now, we need to establish $e\left(T_{m}(B \backslash D),\left\{z_{1}, \ldots, z_{\mathcal{N}}\right\}\right) \leq \varepsilon$. Since $t \in Q \subset H$, we have from (8)

$$
\begin{aligned}
\sup _{x \in T_{m}(B \backslash D)} \min _{t \in Q}\|x-t\|_{1} & \leq \sup _{x \in T_{m}(B \backslash D)} \min _{t \in Q} \min _{h_{0} \in H}\left(\left\|x-h_{0}\right\|_{1}+\left\|h_{0}-t\right\|_{1}\right) \\
& \leq \sup _{x \in T_{m}(B \backslash D)} \min _{t \in Q}\left\|x-h_{0}\right\|_{1}+\sup _{x \in T_{m}(B \backslash D)} \min _{t \in Q} \min _{h_{0} \in H}\left\|h_{0}-t\right\|_{1} \\
& \leq d_{N, \delta}+h .
\end{aligned}
$$

From the inequality (11), the definition of $Q_{\varepsilon^{\prime}}$ and (8), it follows that

$$
\begin{aligned}
e\left(T_{m}(B \backslash D),\left\{z_{1}, \ldots, z_{\mathcal{N}}\right\}\right) & =\sup _{x \in T_{m}(B \backslash D)} \min _{i=1, \ldots, \mathcal{N}}\left\|x-z_{i}\right\|_{1} \\
& \leq \sup _{x \in T_{m}(B \backslash D)} \min _{i=1, \ldots, \mathcal{N}} \min \left(\|x-t\|_{1}+\left\|t-z_{i}\right\|_{1}\right) \\
& \leq \sup _{x \in T_{m}(B \backslash D)} \min _{t \in Q}\left(\|x-t\|_{1}+\min _{i=1, \ldots, \mathcal{N}}\left\|t-z_{i}\right\|_{1}\right) \\
& =\sup _{x \in T_{m}(B \backslash D)} \min _{t \in Q}\|x-t\|_{1}+\varepsilon^{\prime} \\
& \leq d_{N, \delta}+h+\varepsilon^{\prime} \\
& =\varepsilon+h .
\end{aligned}
$$

Consequently, letting $h \rightarrow 0$, we get that $e\left(T_{m}(B \backslash D),\left\{z_{1}, \ldots, z_{\mathcal{N}}\right\}\right) \leq \varepsilon$, which together with (10) completes the proof of Lemma 3.

From the relation (see [28])

$$
\operatorname{vol}\left(B_{p}^{m}\right)=[2 \Gamma(1 / p+1)]^{m} / \Gamma(m / p+1), \quad 1 \leq p \leq \infty,
$$

the balls $B_{p}^{m}$ satisfy the inequalities

$$
\left(c_{p}^{\prime} m\right)^{-m / p}<\operatorname{vol}\left(B_{p}^{m}\right)<\left(c_{p}^{\prime \prime} m\right)^{-m / p}
$$

where $\Gamma$ is the Euler $\Gamma$-function, and $c_{p}^{\prime}, c_{p}^{\prime \prime}$ depend only on $p$.

To estimate $\mathcal{N}_{\varepsilon}\left(T_{m}(B \backslash D)\right)$ from below, we now need another auxiliary result. 
Lemma 4 If $T_{m}$ is a diagonal operator from $\mathbb{R}^{m}$ onto $\ell_{1}^{m}$, then

$$
\left|\operatorname{det}\left(T_{m}\right)\right|=\left|\prod_{i=1}^{m} \lambda_{i}\left(T_{m}\right)\right| \asymp\left(\frac{\left\|T_{m}\right\|}{\sqrt{m}}\right)^{m},
$$

where $\lambda_{i}\left(T_{m}\right), i=1, \ldots, m$, are non-zero eigenvalues of the operator $T_{m}$ rearranged as usual so that $\left|\lambda_{i}\left(T_{m}\right)\right|$ is non-increasing and each eigenvalue is repeated according to its multiplicity.

Proof It is known that

$$
\left\|T_{m}\right\|=\left(\sum_{i=1}^{m}\left(\lambda_{i}\left(T_{m}\right)\right)^{2}\right)^{1 / 2} .
$$

Accordingly,

$$
\sqrt{m}\left|\lambda_{m}\left(T_{m}\right)\right| \leq\left\|T_{m}\right\| \leq \sqrt{m}\left|\lambda_{1}\left(T_{m}\right)\right| .
$$

Obviously,

$$
\left|\lambda_{m}\left(T_{m}\right)\right|^{m} \leq\left|\prod_{i=1}^{m} \lambda_{i}\left(T_{m}\right)\right| \leq\left|\lambda_{m}\left(T_{1}\right)\right|^{m},
$$

from which the result of Lemma 4 follows immediately.

Lemma 5 If $\delta \in[0,1]$ and $\lambda(D) \leq \delta$, then

$$
\mathcal{N}_{\varepsilon}\left(T_{m}(B \backslash D)\right) \geq \frac{1}{3}(1-\delta)\left(c_{0}\left\|T_{m}\right\| / \varepsilon\right)^{m} .
$$

Proof We first establish the inequality

$$
\mathcal{N}_{\varepsilon}\left(T_{m}(B \backslash D)\right) \geq \frac{1}{3} \frac{\lambda\left(T_{m}(B \backslash D)\right)}{\lambda\left(B_{1}^{m}(2 \varepsilon)\right)} .
$$

Indeed, suppose that (13) does not hold. Then, for $\mathcal{N}=\mathcal{N}_{\varepsilon}\left(T_{m}(B \backslash D)\right)$ and some set of points $z_{1}^{*}, \ldots, z_{\mathcal{N}}^{*}$, by

$$
\begin{aligned}
\varepsilon & \geq \sup _{x \in T_{m}(B \backslash D)} \min _{i=1, \ldots, \mathcal{N}}\left\|x-z_{i}^{*}\right\|_{1} \\
& \geq \frac{1}{\lambda\left(T_{m}(B \backslash D)\right)} \int_{T_{m}(B \backslash D)} \min _{i}\left\|x-z_{i}^{*}\right\|_{1} \lambda(d x) \\
& \geq \frac{1}{\lambda\left(T_{m}(B \backslash D)\right)} \int_{T_{m}(B \backslash D) \backslash \bigcup_{i=1}^{\mathcal{N}}\left(z_{i}^{*}+B_{1}^{m}(2 \varepsilon)\right)} \min _{i}\left\|x-z_{i}^{*}\right\|_{1} \lambda(d x) \\
& \geq \frac{1}{\lambda\left(T_{m}(B \backslash D)\right)}(2 \varepsilon) \lambda\left(T_{m}(B \backslash D) \backslash \bigcup_{i=1}^{\mathcal{N}}\left(z_{i}^{*}+B_{1}^{m}(2 \varepsilon)\right)\right) \\
& =2 \varepsilon \frac{\lambda\left(T_{m}(B \backslash D)\right)-\mathcal{N} \cdot \lambda\left(B_{1}^{m}(2 \varepsilon)\right)}{\lambda\left(T_{m}(B \backslash D)\right)}
\end{aligned}
$$




$$
\begin{aligned}
& =2 \varepsilon\left(1-\mathcal{N} \cdot \frac{\lambda\left(B_{1}^{m}(2 \varepsilon)\right)}{\lambda\left(T_{m}(B \backslash D)\right)}\right) \\
& \geq 2 \varepsilon \cdot \frac{2}{3}=\frac{4}{3} \varepsilon
\end{aligned}
$$

we have obtained a contradiction.

In the sequel, we may as well assume that $T_{m}$ is a diagonal operator from $\mathbb{R}^{m}$ onto $\ell_{1}^{m}$. Using the inequality (13), (12) and Lemma 4, we have

$$
\begin{aligned}
\mathcal{N}_{\varepsilon}\left(T_{m}(B \backslash D)\right) & \geq \frac{1}{3} \frac{\lambda\left(T_{m}(B \backslash D)\right)}{\lambda\left(B_{1}^{m}(2 \varepsilon)\right)} \\
& =\frac{1}{3} \frac{\left|\operatorname{det}\left(T_{m}\right)\right| \lambda(B \backslash D)}{\lambda\left(B_{1}^{m}(2 \varepsilon)\right)} \\
& \geq \frac{1}{3} c\left(\frac{\left\|T_{m}\right\|}{\sqrt{m}}\right)^{m}(1-\delta) \frac{\operatorname{vol}(B)}{\operatorname{vol}\left(B_{1}^{m}(2 \varepsilon)\right)} \\
& \geq \frac{1}{3}\left(\frac{c_{1}\left\|T_{m}\right\|}{\sqrt{m}}\right)^{m}(1-\delta)\left(\frac{c_{2} \sqrt{m}}{2 \varepsilon}\right)^{m} \\
& =\frac{1}{3}(1-\delta)\left(\frac{c_{0}\left\|T_{m}\right\|}{\varepsilon}\right)^{m} .
\end{aligned}
$$

Next, assume that $T_{m}$ is a symmetric transformation of $\mathbb{R}^{m}$, then there is an orthogonal matrix $U$ of order $m$ such that the matrix $U T_{m} U^{T}$ is a diagonal matrix. Since the Lebesgue measure is invariant for orthogonal transformation, the result holds for symmetric transformation $T_{m}$.

Finally, in the general case, $T_{m}$ is a general invertible linear transformation from $\mathbb{R}^{m}$ onto $\ell_{1}^{m}$, then there are two matrices $U$ and $S$ such that $T_{m}=U S$, where $U$ is an orthogonal matrix and $S$ is a positive definite symmetric matrix. As the same reason above, the result holds for the transformation $T_{m}$.

Thus, we complete the proof of Lemma 5.

Lemma 6 If $\delta \in[0,1 / 2]$, then

$$
d_{N, \delta}\left(T_{m}: B \rightarrow \ell_{1}^{m}, \lambda\right) \geq c_{1}\left\|T_{m}\right\|
$$

Proof From Lemma 3 and Lemma 5, we get

$$
c\left(1+\frac{4\left\|T_{m}\right\|}{\varepsilon-d_{N, \delta}}\right)^{m} \geq N_{\varepsilon}\left(T_{m}(B \backslash D)\right) \geq \frac{1}{3}(1-\delta)\left(\frac{c_{0}\left\|T_{m}\right\|}{\varepsilon}\right)^{m} \geq \frac{1}{6}\left(\frac{c_{0}\left\|T_{m}\right\|}{\varepsilon}\right)^{m} .
$$

Let $\varepsilon=5 d_{N, \delta}$. Taking the logarithm of the inequality (14), we get the inequality

$$
d_{N, \delta}\left(T_{m}: B \rightarrow \ell_{1}^{m}, \lambda\right) \geq c_{1}\left\|T_{m}\right\|
$$

for some constants $c_{1}$ and $c_{2}$ and $N$ with $m \geq c_{2} N$. Lemma 6 is proved.

Proof of Theorem 2 According to Lemma 6 , for any $\delta \in[0,1 / 2]$ and any subspace $\mathcal{L} \subset \mathbb{R}^{m}$ with $\operatorname{dim} \mathscr{L} \leq N$, there is a set $K \subset B$ with Lebesgue measure $\lambda(K)>\delta$ such that

$$
e\left(T_{m} x, \mathcal{L}, \ell_{1}^{m}\right) \geq c_{1}\left\|T_{m}\right\|
$$


for any element $x \in K$. On the unit sphere $S^{m-1}=\left\{x \in \mathbb{R}^{m}:\|x\|_{2}=1\right\}$, we consider the subset $K^{\prime}=\left\{x /\|x\|_{2}: x \in K\right\}$.

Let $\lambda_{S^{m-1}}$ be a Lebesgue measure on the sphere $S^{m-1}$. We prove that

$$
\lambda_{S^{m-1}}\left(K^{\prime}\right)>\delta \lambda_{S^{m-1}}\left(S^{m-1}\right) .
$$

Indeed, assume that

$$
\lambda_{S^{m-1}}\left(K^{\prime}\right) \leq \delta \lambda_{S^{m-1}}\left(S^{m-1}\right) .
$$

We introduce in $\mathbb{R}^{m}$ a polar system of coordinates $(r, s)$, where $r \geq 0$ and $s \in S^{m-1}$, and consider in $B$ the cone

$$
C=\left\{(r, s): 0 \leq r \leq 1, s \in K^{\prime}\right\}
$$

Then

$$
\begin{aligned}
\lambda(K) & \leq \lambda(C)=\frac{1}{\operatorname{vol}(B)} \int_{0}^{1} r^{m-1} d r \int_{K^{\prime}} \lambda_{S^{m-1}}(d s) \\
& \leq \frac{\delta}{\operatorname{vol}(B)} \int_{0}^{1} r^{m-1} d r \int_{S^{m-1}} \lambda_{S^{m-1}}(d s)=\delta .
\end{aligned}
$$

We have obtained a contradiction.

Consider the set $K_{t}=\left\{(r, s): r \geq t, s \in K^{\prime}\right\}, t \geq 0$. Using the inequality (16), we estimate the Gaussian measure of $K_{t}$ :

$$
\begin{aligned}
v\left(K_{t}\right) & =(2 \pi)^{-m / 2} \int_{K_{t}} \exp \left(-\frac{1}{2}\|u\|_{2}^{2}\right)(d u) \\
& =(2 \pi)^{-m / 2} \int_{t}^{\infty} r^{m-1} \exp \left(-\frac{r^{2}}{2}\right) d r \int_{K^{\prime}} \lambda_{S^{m-1}}(d s) \\
& \geq(2 \pi)^{-m / 2} \delta \int_{t}^{\infty} r^{m-1} \exp \left(-\frac{r^{2}}{2}\right) d r \int_{S^{m-1}} \lambda_{S^{m-1}}(d s) \\
& =\delta v\left(x:\|x\|_{2} \geq t\right) .
\end{aligned}
$$

A direct computation shows that for $t \geq \sqrt{m}$,

$$
v\left(\|x\|_{2} \geq t\right) \geq \exp \left(-t^{2}\right) \quad \text { and } \quad v\left(\|x\|_{2} \geq \sqrt{m}\right) \geq c_{0}>0
$$

where $c_{0}$ is some absolute constant. It follows from this and (17) that for $t_{0}=\max \{\sqrt{m}$, $\sqrt{\ln (1 / \delta)}\}$ and for any $\delta \in\left(0, c_{0}\right]$,

$$
v\left(K_{t_{0}}\right) \geq \delta \cdot v\left(\|x\|_{2}>\max \{\sqrt{m}, \sqrt{\ln (1 / \delta)}\}\right)>\delta^{2} .
$$

For any element $y=r s \in K_{t_{0}}$, we have from (15)

$$
\begin{aligned}
e\left(T_{m} y, \mathcal{L}, \ell_{1}^{m}\right) & =\operatorname{re}\left(T_{m} s, \mathcal{L}, \ell_{1}^{m}\right) \geq c_{1} r\left\|T_{m}\right\| \geq c_{2}\left\|T_{m}\right\| \max \{\sqrt{m}, \sqrt{\ln (1 / \delta)}\} \\
& \geq \frac{c_{2}}{2}\left\|T_{m}\right\| \sqrt{m+\ln (1 / \delta)} .
\end{aligned}
$$


Since $\mathscr{L}$ is an arbitrary subspace with $\operatorname{dim} \mathscr{L} \leq N$, it follows from (18) and (19) that

$$
d_{N, \delta^{2}}\left(T_{m}: \mathbb{R}^{m} \rightarrow \ell_{1}^{m}, v\right) \geq \frac{c_{2}}{2}\left\|T_{m}\right\| \sqrt{m+\ln (1 / \delta)} .
$$

Theorem 2 is a direct consequence of this.

\section{Competing interests}

The authors declare that they have no competing interests.

\section{Authors' contributions}

Both authors completed the paper together. They also read and approved the final manuscript.

\section{Author details}

${ }^{1}$ Hulunbeier Vocational and Technological College, Hulunbeier, Inner Mongolia 021000, China. ${ }^{2}$ School of Mathematical Sciences, Hulunbeier University, Hulunbeier, Inner Mongolia 021008, China.

\section{Acknowledgements}

The authors thank the editor and the referees for their valuable suggestions to improve the quality of this paper. The present investigations was supported by the Natural Science Foundation of Inner Mongolia Province of China under Grant 2011 MS0103.

\section{Received: 20 October 2012 Accepted: 15 May 2013 Published: 3 June 2013}

\section{References}

1. Sul'din, P: Wiener measure and its applications to approximation theory I. Izv. Vysš. Učebn. Zaved., Mat. 6, 145-158 (1959) (in Russian)

2. Sul'din, P: Wiener measure and its applications to approximation theory II. Izv. Vysš. Učebn. Zaved., Mat. 5, 165-179 (1960) (in Russian)

3. Traub, JF, Wasilkowski, GW, Woźniakowski, H: Information-Based Complexity. Academic Press, New York (1988)

4. Maiorov, VE: Discretization of the problem of diameters. Usp. Mat. Nauk 30, 179-180 (1975)

5. Maiorov, VE: On linear widths of Sobolev classes and chains of extremal subspaces. Mat. Sb. 113(115), 437-463 (1980). English transl. in Math. USSR Sb. 41 (1982)

6. Maiorov, VE: Kolmogorov's $(N, \delta)$-widths of the spaces of the smooth functions. Russ. Acad. Sci. Sb. Math. 79, 265-279 (1994)

7. Maiorov, VE: Linear widths of function spaces equipped with the Gaussian measure. J. Approx. Theory 77, 74-88 (1994)

8. Mathé, P. S-Numbers in information-based complexity. J. Complex. 6, 41-66 (1990)

9. Mathé, P: Random approximation of Sobolev embeddings. J. Complex. 7, 261-281 (1991)

10. Mathé, P: A minimax principle for the optimal error of Monte Carlo methods. Constr. Approx. 9, 23-29 (1993)

11. Mathé, P: On optimal random nets. J. Complex. 9, 171-180 (1993)

12. Mathé, P: Approximation theory of stochastic numerical methods. Habilitationsschrift, Fachbereich Mathematik, Freie Universität Berlin, Berlin (1994)

13. Sun, $Y$, Wang, $C: \mu$-Average $n$-widths on the Wiener space. J. Complex. 10, 428-436 (1994)

14. Sun, Y, Wang, C: Average error bounds of best approximation of continuous functions on the Wiener space. J. Complex. 11, 74-104 (1995)

15. Ritter, K: Average-Case Analysis of Numerical Problems. Lecture Notes in Math., vol. 1733. Springer, New York (2000)

16. Fang, G, Qian, L: Approximation characteristics for diagonal operators in different computational settings. J. Approx. Theory 140, 178-190 (2006)

17. Fang, G, Qian, L: Optimization on class of operator equations in the probabilistic case setting. Sci. China Ser. A 50 100-104 (2007)

18. Fang, G, Ye, P: Probabilistic and average linear widths of Sobolev space with Gaussian measure. J. Complex. 19, 73-84 (2003)

19. Fang, G, Ye, P: Probabilistic and average linear widths of Sobolev space with Gaussian measure in $L_{\infty}$ norm. Constr. Approx. 20, 159-172 (2004)

20. Chen, G, Fang, G: Probabilistic and average widths of multivariate Sobolev spaces with mixed derivative equipped with the Gaussian measure. J. Complex. 20, 858-875 (2004)

21. Chen, G, Fang, G: Linear widths of multivariate function spaces equipped with the Gaussian measure. J. Approx. Theory 132, 77-96 (2005)

22. Carl, B, Pajor, A: Gelfand numbers of operators with values in a Hilbert space. Invent. Math. 94, $479-504$ (1988)

23. Pinkus, A: $n$-Widths in Approximation Theory. Springer, New York (1985)

24. Carl, B, Stephani, I: Entropy, Compactness and the Approximation of Operators. Cambridge University Press, Cambridge (1990)

25. Pietsch, A: Operator Ideals. North-Holland, Amsterdam (1980)

26. Pietsch, A: Eigenvalues and s-Numbers. Geest und Portig, Leipzig (1987)

27. Gluskin, ED: Norm of random matrices and widths of finite-dimensional sets. Mat. Sb. 120(162), 180-189 (1983). English transl. in Math. USSR Sb. 48 (1984)

28. Pisier, G: The Volume of Convex Bodies and Banach Space Geometry. Cambridge University Press, Cambridge (1989) 
doi:10.1186/1029-242X-2013-277

Cite this article as: Zhou and Li: Estimates of probabilistic widths of the diagonal operator of finite-dimensional sets with the Gaussian measure. Journal of Inequalities and Applications 2013 2013:277.

Submit your manuscript to a SpringerOpen ${ }^{\circ}$ journal and benefit from:

- Convenient online submission

- Rigorous peer review

- Immediate publication on acceptance

- Open access: articles freely available online

- High visibility within the field

- Retaining the copyright to your article

Submit your next manuscript at $\gg$ springeropen.com 